\title{
Brain-Derived Neurotrophic Factor Administration Mediated Oligodendrocyte Differentiation and Myelin Formation in Subcortical Ischemic Stroke
}

Short title: BDNF-Induced White Matter Repair in Stroke

Authors: Jaime Ramos-Cejudo, BiolD, ${ }^{1 *}$ María Gutiérrez-Fernández, $\mathrm{PhD},{ }^{1 *}$ Laura Otero-Ortega, $\mathrm{PhD},{ }^{1 *}$ Berta Rodríguez-Frutos, $\mathrm{PhD},{ }^{1}$ Blanca Fuentes, $\mathrm{PhD},{ }^{1}$ Maria Teresa Vallejo-Cremades, PhD, ${ }^{1}$ Teresa Navarro Hernanz, BiolD, ${ }^{2}$ Sebastián Cerdán, $\mathrm{PhD},{ }^{2}$ Exuperio Díez-Tejedor PhD F.A.H.A F.E.S.O ${ }^{1 \square}$

\section{*Contributed equally}

Affiliations:

${ }^{1}$ Department of Neurology and Stroke Center, Neuroscience and Cerebrovascular Research Laboratory, La Paz University Hospital, Neuroscience Area of IdiPAZ Health Research Institute, Autónoma University of Madrid, Madrid, Spain

${ }^{2}$ Laboratory for Imaging and Spectroscopy by Magnetic Resonance (LISMAR), Institute of Biomedical Research Alberto Sols, CSIC-UAM, Madrid, Spain

\section{$\square$ Corresponding author:}

Prof. Exuperio Díez-Tejedor, MD, PhD, FAHA, FESO

María Gutiérrez-Fernández, PhD

Department of Neurology and Stroke Center

Neuroscience and Cerebrovascular Research Laboratory

La Paz University Hospital

IdiPAZ Health Research Institute

Autónoma University of Madrid

Paseo de la Castellana 261, 28046, Madrid, Spain

e-mail: exuperio.diez@salud.madrid.org,mariagutierrezfdez@hotmail.com 


\begin{abstract}
Background and Purpose-Translational research is beginning to reveal the importance of trophic factors as a therapy for cellular brain repair. The purpose of this study was to analyze whether brain-derived neurotrophic factor (BDNF) administration could mediate oligodendrogenesis and remyelination after white matter injury in subcortical stroke.
\end{abstract}

Methods-Ischemia was induced in rats by injection of endothelin- 1 . At $24 \mathrm{~h}, 0.4 \mu \mathrm{g} / \mathrm{kg}$ of BDNF or saline was intravenously administered to the treatment and control groups, respectively. Functional evaluation, magnetic resonance imaging (MRI) and fiber tract integrity on tractography images were analyzed. Proliferation (KI-67) and white matter repair markers (A2B5, CNPase, APC, PDGFR-alpha, O4,Olig-2 and MBP) were analyzed at $7 \mathrm{~d}$ and $28 \mathrm{~d}$.

Results-The BDNF-treated animals showed less functional deficit at $28 \mathrm{~d}$ after treatment than the controls $(\mathrm{p}<.05)$. Although T2-MRI did not show differences in lesion size at $7 \mathrm{~d}$ and $28 \mathrm{~d}$ between groups, diffusion tensor imaging (DTI) tractography analysis revealed significantly better tract connectivity at $28 \mathrm{~d}$ in the BDNF group than in the controls $(\mathrm{p}<.05)$. Increased proliferation of oligodendrocyte progenitors was observed in treated animals at $7 d(p<.05)$. Finally, the levels of white matter repair markers $(A 2 B 5$, CNPase, O4 at 7d; Olig-2, MBP at 28d) were higher in the BDNF group than in the controls $(\mathrm{p}<.05)$.

Conclusions-BDNF administration exerted better functional outcome, oligodendrogenesis, remyelination and fiber connectivity than controls in rats subjected to subcortical damage in ischemic stroke. 
Keywords: BDNF, White matter injury, Subcortical stroke, Brain repair, Endothelin-1.

\section{Background}

After decades of research focused on the search for a treatment for cortical infarcts in experimental models in which the gray matter is most affected, a few translational studies are beginning to highlight the importance of considering the white matter component after stroke. ${ }^{1}$ Not only are up to $25 \%$ of ischemic strokes in humans subcortical or lacunar and confined to white matter regions ${ }^{2}$ but cortical infarcts also produce white matter injury. The high frequency of this damage motivates the search for an effective therapy to enhance the mechanisms underlying the repair of damaged white matter (axons and myelin) after a stroke. ${ }^{2}$

Trophic factors are emerging as a viable repair therapy in stroke, and they can strongly promote a favorable environment for cellular repair after brain injury. ${ }^{3,4}$ One of the prominent trophic molecules is brain-derived neurotrophic factor (BDNF), which is secreted in an activity-dependent manner and crucially promotes synaptic regulation and axonal plasticity associated with learning, memory and sensorimotor recovery., ${ }^{4,5}$ Furthermore, in vitro and BDNF knock-out studies have demonstrated that this trophic factor has direct effects on oligodendroglia, promoting the proliferation and differentiation of oligodendrocyte precursor cells (OPC) and myelination. ${ }^{6-8}$

All of the above indicate that BDNF could exert a possible effect upon oligodendrogenesis and remyelination after a stroke. Thus, the present study explored the possible effect of BDNF administration on white matter remodeling via oligodendrogenesis and myelinogenesis and whether this effect might correlate with functional recovery in an animal model of subcortical stroke. 


\section{Materials and Methods}

\section{Study design}

This translational study followed all STAIR (stroke therapy academic industry roundtable) and RIGOR guidelines in terms of randomization, blinding and statistical power. ${ }^{9}$ In addition, the experiments were designed to minimize animal suffering in compliance with our medical school's Ethical Committee for the Care and Use of Animals in Research (EU directives 86/609/CEE and 2003/65/CE). A total of 74 adult Sprague-Dawley rats (200 to $250 \mathrm{~g}$ ) were randomly distributed into 3 groups: Sham: surgery without endothelin-1 (ET-1) injection + IV saline administration ( $n=24)$; Control: subcortical stroke + IV saline administration $(n=24)$; and BDNF animals: subcortical stroke + IV BDNF treatment $(n=24)$. In the BDNF group, recombinant human BDNF (Peprotech,UK) was diluted in saline to a final volume of $1 \mathrm{~mL}$ and administered through the tail vein at a final dose of $0.4 \mu \mathrm{g} / \mathrm{kg}$ at $24 \mathrm{~h}$ after surgery. A saline solution of the same volume was delivered to the control and sham animals. The rats were then randomly divided into 3 subgroups that were euthanized at $4 \mathrm{~h}(\mathrm{n}=4 / \mathrm{each}$ group), at $7 \mathrm{~d}(\mathrm{n}=10 /$ each group) or at $28 \mathrm{~d}(\mathrm{n}=10 /$ each group). Two rats were excluded from the study because one died after surgery and the other died during the magnetic resonance procedure.

\section{ET-1 subcortical stroke model}

In all the animals, physiological parameters and body temperature were continuously monitored during surgery (Online-only Data Supplement). To provoke white matter injury, a subcortical stroke was induced to pre-anesthetized rats following a small craniotomy by the injection of a potent vasoconstrictor, endothelin-1, using a 
stereotactic apparatus employing stereotactic references $(+0.4 \mathrm{mmAP},+3.5 \mathrm{mmL}$, $+6 \mathrm{mmDV}$ from the bregma). $1 \mu \mathrm{L}$ of ET-1 $(0.25 \mathrm{ug} / \mu \mathrm{L})$ was delivered at a final speed of $0.2 \mu \mathrm{L} / \mathrm{m}$. Immediately after surgery, analgesia was provided to all groups by an intraperitoneal injection of meloxicam at $2 \mathrm{mg} / \mathrm{Kg}$.

\section{BDNF quantification after treatment}

BDNF was quantified by the human BDNF ELISA kit (ABCAM) and Western blot in brain tissue and serum in both the control and treated animals at $4 \mathrm{~h}, 7$ and $28 \mathrm{~d}$ (Onlineonly Data Supplement). Immunofluorescence (using a primary antibody anti-BDNF [1:1000, Millipore] and a secondary antibody anti-rabbit Alexa Fluor 594 [1:750, Invitrogen]) ( $\mathrm{n}=4$ per group).

\section{Functional evaluation scales}

Functional evaluation was scored in all animals by a blinded observer before surgery and at 1, 3, 7 and 28d after treatment. Motor performance evaluation was measured by beam walking test, ${ }^{10}$ the rotarod test ${ }^{11}$ and the Modified Rogers Scale ${ }^{12}$ (Online-only Data Supplement).

\section{In vivo analysis by MRI and DTI tractography}

To analyze whether the BDNF effect could be examined with in vivo imaging techniques, we studied the ipsilateral hemisphere at the site of the ET-1-induced lesion using T2-MRI, diffusion on ADC maps and tract connectivity using DTI tractography ( $n=6$ of each group) at 7 and 28d after treatment (Online-only Data Supplement). 


\section{Cell proliferation analysis}

KI-67 staining was analyzed on days 7 and 28, using 10 sections selected from each animal ( $\mathrm{n}=6$ animals per group) (Online-only Data Supplement).

To evaluate differentiation markers in proliferating cells, we double stained KI-67positive cells with CNPase, A2B5, APC and PDGFR-alpha by immunofluorescence. All the images were acquired as a maximum confocal projection.

\section{Immunohistochemistry, immunofluorescence and Western blot}

CryoMyelin staining kit (Hito Biotech) that allows sensitive localization and visualization of the myelin fibers was performed on frozen sections. The mean region of interest (ROI) intensity in the CryoMyelin staining was quantified using a Nikon Eclipse-Ti inverted microscope and NIS-elements software. The lesion zone was studied in more detail using immunofluorescence and Western blot as previously described ( $\mathrm{n}=4$ animals of each group). ${ }^{11}$ CNPase, A2B5, O4, Nogo-A, MBP and Olig2 markers were studied at 7 and 28d (Online-only Data Supplement).

\section{Statistical analysis}

The data are presented as mean \pm standard error of the mean (SEM). The KruskalWallis test followed by the Mann-Whitney U test was used to compare data. Values of $\mathrm{p}<.05$ were considered significant at a $95 \%$ confidence interval $(\mathrm{CI})$; the data were calculated using statistical software programs SPSS 16 and GraphPad. 


\section{Results}

\section{BDNF levels were increased after treatment}

ELISA analysis of serum BDNF showed that the levels were augmented at $4 \mathrm{~h}$ after its intravenous administration (4.33 $\pm 0.32 ; 5.61 \pm 0.62 ; \mathrm{p}<.05)$ (Fig. 1A). BDNF was analyzed in the BDNF-treated group and in the control animals throughout the brain. At 4h, significant higher levels of BDNF were found in the area of injury in BDNF animals $(6.67 \pm 0.43)$ compared with the controls $(0.79 \pm 0.24)(\mathrm{p}<.05)$. Also Western blot analysis detected higher levels of BDNF in the ischemic lesion in the treated animals $(0.97 \pm 0.08)$ compared with the control group $(0.79 \pm 0.24)$ at 4 h after treatment $(\mathrm{p}<.05)$. At 7 and 28d after administration, the BDNF levels were not significantly different in the treated animals $(0.28 \pm 0.03$ and $0.20 \pm 0.08)$ compared with the control group $(0.27 \pm 0.063$ and $0.204 \pm 0.049)$, respectively (Fig.1B,1C). Immunofluorescence for BDNF showed both intracellular and extracellular staining (Fig.1C).

\section{BDNF improved functional recovery in the subcortical white matter injury model}

No significant differences were found in the functional outcome of the treated and control animals at 1, 3 and $7 \mathrm{~d}$ after treatment. However, compared with the control rats, $28 \mathrm{~d}$ after treatment the BDNF treated rats showed significantly better rotarod test performances $(67.25 \pm 25.10$ and $103.00 \pm 16.10$, respectively; $\mathrm{p}=.049)$, beam walking (3.5 \pm 0.70 and 1.4 \pm 0.54 , respectively; $\mathrm{p}=.042)$ and Modified Rogers Test $(2.5 \pm 0.57$ and 1.2 \pm 0.44 , respectively; $\mathrm{p}=.018)$ (Fig.2A). 
BDNF effects on white matter were negligible by in vivo MRI but perceptible by tractography and myelin staining

The lesion size viewed on MRI in the BDNF-treated animals was indistinguishable from the control group at $7 \mathrm{~d}(7.30 \pm 2.56$ and $14.75 \pm 1.72$, respectively) and $28 \mathrm{~d}$ (7.49 \pm 1.79 and 8.77 \pm 3.97 , respectively) after treatment (p>.05) (Fig.2C).

DTI tractography data showed similar results in axial diffusivity $(124.297 \pm 10.72$ and $133.73 \pm 14.497 ; \mathrm{p}>.05)$ and diffusion in ADC maps (93.58 \pm 10.033 and $123.307 \pm 23.839$; $\mathrm{p}>.05$ ) in both the control and treated groups, respectively, at 7d after stroke. However, compared with the control rats, 28d after treatment the BDNF-treated rats showed significantly improved axial diffusivity $(126.39 \pm 7.82$ and $159.715 \pm 14.761$, respectively; $\mathrm{p}<.05)$ and diffusion in ADC maps $(129.743 \pm 11.934$ and 157.926 \pm 12.562 , respectively; $\mathrm{p}<.05)($ Fig.2C). This result suggests that there was a significant improvement in white matter thickness (width, breadth, depth) and restoration of tract connectivity in the BDNF-treated animals compared with controls at $28 \mathrm{~d}$.

These results are in agreement with the morphological study of myelin fibers by CryoMyelin staining. The mean ROI intensity was calculated at the lesion site in the control and BDNF-treated animals, in which white intensity indicated absence of myelin and black intensity the presence of myelinated axons. The results showed higher intensity (absence of axons) in controls (198.61 \pm 26.30$)$ compared with BDNF-treated animals (172.18 \pm 17.40$)$ (Fig.2B). 


\section{BDNF administration enhances OPC proliferation after white matter injury}

Numerous KI-67-positive cells were observed in the ischemic lesion in the control animals and in the BDNF-treated group. The number of KI-67-positive cells $(170 \pm 17.32)$ was significantly higher in the ischemic lesion compared with control group (34.25 \pm 18.28$)(\mathrm{p}=.032) 7 \mathrm{~d}$ after BDNF treatment (Fig.3A).

Double staining of KI-67-positive cells with the OPC markers was observed to be higher in the subventricular zone in treated animals than in controls for A2B5 $(9.12 \pm 1.87 \%$ and $5.45 \pm 2.10 \%)$, CNPase $(31.00 \pm 7.32 \%$ and $22.12 \pm 5.34 \%)$, APC $(26.00 \pm 5.12 \%$ and $17.32 \pm 4.21 \%)$ and PDGFR-alpha $(36.00 \pm 6.23 \%$ and $28.00 \pm 7.34 \%)$, suggesting that OPC proliferation is enhanced after BDNF injection (Fig.3B).

\section{BDNF injection increases OPC markers 7d after axonal disruption}

The levels of OPC markers were analyzed by Western blot in the lesion area at 7 and 28d after treatment (Fig.4B). There was a significant increase in CNPase marker levels in the BDNF-treated animals $(217.00 \pm 14.10)$ compared with the controls at $7 d$ $(183.20 \pm 16.32) \quad(\mathrm{p}<.05)$. A2B5 was also higher in the BDNF-treated animals $(141.20 \pm 41.20)$ than in the controls $(75.39 \pm 13.20)$ at $7 \mathrm{~d}$ after treatment $(\mathrm{p}<.05)$. We also found higher levels $\mathrm{O} 4$ marker at $7 \mathrm{~d}$, in the BDNF-treated group $(190.88 \pm 20.19)$ than in the control animals $(82.10 \pm 18.01 ; \mathrm{p}<.05)$. At $28 \mathrm{~d}$ the levels of OPC markers were lower in both control and BDNF animals. Also, when comparing treated and control groups at 28d, no significant differences were found (A2B5 [91.12 \pm 41.10 vs 112.10 \pm 13.81 , CNPase [89.23 \pm 21.10 vs $110.10 \pm 21.30]$ and O4 [75.20 \pm 19.10 vs $69.60 \pm 16.13]$ respectively). IHF analysis confirmed these results (Fig.4A). 
BDNF administration enhances oligodendrocyte maturation and axonal growthassociated markers $28 d$ post injury

At 7 and 28d we analyzed markers related to later developmental steps in white matter differentiation (MBP, Olig-2) and in maturation of myelin fibers (Nogo-A) (Fig.5A,5B).

Western blot analysis showed significantly higher levels of the oligodendrocyte marker Olig-2 in the BDNF-treated animals than in the control group at $28 \mathrm{~d}$ $(242.85 \pm 15.50$ and $94.21 \pm 10.20$, respectively; $p<.05)$ and a significant increase in MBP in the treated animals compared with the controls $(192.18 \pm 29.99$ and $106.55 \pm 26.74$, respectively; $\mathrm{p}<.0 .05)$. We also found a significant decrease in Nogo-A levels in the BDNF-treated animals compared with the controls at $7 \mathrm{~d}(160.11 \pm 17.23$ and $213.21 \pm 12.41$ respectively) and at $28 \mathrm{~d}(163.01 \pm 18.88$ and $200.10 \pm 9.87$, respectively; $\mathrm{p}=.014)$. At $7 \mathrm{~d}$ the levels of Olig-2 (150.25 \pm 19.43 and $70.91 \pm 21.50)$ and MBP $(51.21 \pm 23.10$ and $25.01 \pm 10.80)$ were too low compared with $28 \mathrm{~d}$ in both the treated group and control group because all these markers are related to later developmental steps in white matter differentiation. 


\section{Discussion}

In the current search for new therapeutic strategies to improve functional and cognitive deficits after stroke, it is worth remembering that myelination failure prevention is necessary for brain repair processes. Thus, the present study employed an intravenous infusion $(0.4 \mu \mathrm{g} / \mathrm{kg})$ of BDNF as a therapeutic strategy to prevent myelination failure. The group treated with BDNF injection showed improved functional recovery and a significant increase in the number of proliferating cells, including OPC, after white matter injury. After using BDNF, large numbers of cells expressed OPC markers such as CNPase, A2B5 and $\mathrm{O} 4$ at $7 \mathrm{~d}$. At 28d after treatment, the cells began to acquire specific markers of oligodendrocyte differentiation, such as Olig-2 and MBP, suggesting that repair of white matter fiber tracts was induced by the BDNF injection. The results support a role for BDNF in improving the repair of white matter and in OPC proliferation and differentiation in this experimental subcortical stroke model.

This treatment was chosen because systemic administration is already known to allow BDNF to cross the blood brain barrier. ${ }^{4,13}$ In this sense, the BDNF levels in our study were augmented in brain tissue and peripheral serum at $4 \mathrm{~h}$ after injection. This increase of BDNF levels observed in the brain might enhance recovery mechanisms after stroke.

BDNF as therapy to induce brain protection and brain repair is becoming more common in translational research. After a cortical stroke, BDNF has been shown to control neuronal circuits, increase the number of newborn neurons in several brain areas, ${ }^{3,4}$ reduce astrogliosis, enhance axonal growth in the ischemic border zone ${ }^{5}$ and stimulate the plasticity of dendritic branching and synaptic transmission. ${ }^{14}$ However, the effects of BDNF administration on nerve fiber repair, myelin formation and remodeling after white matter injury are still unknown. 
In a translational study it is important to analyze whether BDNF injections act on the motor dysfunctions that are characteristic to subcortical stroke. Previous authors found the beam walking test, ${ }^{10}$ the rotarod test ${ }^{11}$ and the Modified Rogers Test ${ }^{12}$ to be effective in assessing the motor deficit associated primarily with subcortical stroke. In this study, BDNF treatment induced a significant improvement in functional recovery that was particularly notable at $28 \mathrm{~d}$ after treatment compared with the controls. These results clearly suggest a true recovery-enhancing effect for BDNF. Although there are no previous studies that have administered BDNF in a subcortical stroke model, our results are consistent with previous data showing a better functional outcome in animals treated intravenously with BDNF after cortical ischemia. ${ }^{4}$

Focal injection of the vasoconstrictor ET-1 into the subcortical white matter produces a visible infarct on MRI, as previously shown in subcortical stroke studies. ${ }^{15,16}$ To analyze whether the BDNF effect could be visualized with in vivo imaging techniques, we performed T2-MRI, ADC maps and DTI tractography. No significant differences in lesion size were observed in the BDNF-treated animals compared with the control group on the T2-MRI images; however, analyzing fiber tract integrity by DTI tractography showed that tract thickness was recovered at $28 \mathrm{~d}$ after BDNF administration. This result suggests that functional recovery at $28 \mathrm{~d}$ could be related to the process by which restructured axons, which had previously been compromised and demyelinated, recover not only their proper structure but also tract connectivity. The lack of a relationship between the T2-MRI and DTI tractography images, however, remains unclear. This is why we intensified the histological analysis of the injured tissue in this study. 
After a stroke, myelin injury activates the OPC distributed throughout the white matter, which proliferate and migrate to the site of damage where they subsequently mature into myelinating oligodendrocytes that ensheath axons to form the myelin membrane. ${ }^{1,17}$ Our study using colocalization of the KI-67 antigen and the CNPase, A2B5, APC and PDGFR-alpha markers showed BDNF to be a potent factor for enhancing the OPC proliferation response after white matter injury. Our findings agree with other non-stroke studies in vitro and in BDNF knock-out mice, showing that BDNF exerts direct effects upon oligodendroglia, promoting OPC proliferation and differentiation, as well as myelination. ${ }^{6-8}$ To elucidate whether BDNF administration also acts on oligodendrocytes and shattered white matter fibers and increases some repair mechanisms, including nerve fiber remodeling, axonal sprouting, oligodendrogenesis and myelinogenesis, we measured white matter repair-associated markers in both the treated and the control animals. Various white matter repair markers were studied at two different time points, $7 \mathrm{~d}$ and $28 \mathrm{~d}$. Related to the first steps of the genesis and migration of white matter progenitor cells, markers such as A2B5 (a characteristic OPC marker), $\mathrm{O} 4$ and CNPase (markers related to immature oligodendrocytes), were studied in the lesion area. Markers related to white matter differentiation and myelin fiber maturation such as MBP (myelin marker), Olig-2 (mature oligodendrocytes) and the myelin inhibitor Nogo-A were also analyzed.

The best-characterized oligodendroglial progenitor marker is A2B5. ${ }^{18,19}$ This molecule is a cell surface ganglioside expressed on developing oligodendroglial progenitors. In our study, the levels of the A2B5 marker were higher in the BDNF-treated animals at 7d than in the control group. $\mathrm{O} 4$ is another marker expressed in pro-oligodendrocytes during oligodendrocyte differentiation. ${ }^{20}$ Our study found an increase in the amount of 
O4 protein in the animals subjected to BDNF administration compared with their controls. This observation agrees with a study describing the levels of CNPase. ${ }^{19}$ The presence of CNPase appears to be one of the earliest events in oligodendrocyte differentiation and BDNF-treated animals showed higher levels of this marker than the control group. The levels of A2B5, CNPase and O4 were too low at 28d because all these markers are related to early events in oligodendrocyte differentiation. These results are in line with previous non-stroke in vitro studies in which the effects of BDNF are examined in oligodendrocyte progenitors, finding enhanced A2B5 and O4 expression after neurotrophin administration..$^{21}$

Regarding the oligodendrocyte maturation-associated proteins, the levels of these markers were found to be too low at $7 \mathrm{~d}$. However, Olig-2 levels were elevated in the treated animals compared with the control animals at $28 \mathrm{~d}$, an observation that could be explained by BDNF having enhanced oligodendroglial cell differentiation. However, both the presence of mature oligodendrocytes and coating the myelin sheath are important for white matter repair. There was a significant increase of MBP reactivity in the white matter tracts at $28 \mathrm{~d}$ in the treated animals compared with the control group. Although there are no previous studies that relate BDNF to remyelination after stroke, these increases in the levels of MBP and Olig-2 concur quite well with previous nonstroke studies indicating that BDNF influences differentiating oligodendrocytes by increasing both the number of MBP cells and the expression of the MBP protein. ${ }^{22}$ What is unknown is whether the increased myelination was because more axons were being myelinated or whether there was an overall increase in myelin thickness.

Among the myelin-associated proteins, Nogo-A has been shown to be particularly powerful in preventing axonal growth and plasticity. ${ }^{23}$ Nogo-A not only inhibits axonal 
growth, but also prevents neurotrophins such as BDNF from binding to this receptor inhibiting axonal growth. ${ }^{24}$ Some studies have demonstrated that inhibition mediated by Nogo-A is blocked if neurons are exposed to BDNF before encountering the inhibitor. ${ }^{25}$ The present study concurs with these observations because at $28 \mathrm{~d}$ after treatment there was a significant decrease in the levels of Nogo-A in BDNF animals. These results indicate that BDNF injection could enhance axonal growth and plasticity by decreasing Nogo-A levels. The observations found at $28 \mathrm{~d}$ suggest that functional recovery might be related to the axonal sprouting subsequent to BDNF administration, and could be indicative of the process by which growing and as-yet demyelinated axons are recovered with new myelin sheaths. Furthermore, this interpretation of these observations agrees with the tractography images that suggest that tract connectivity was being restored in the infarcted area at the same time point.

\section{Conclusions}

Our study helped us to identify a clear role for BDNF in improving functional outcome by mediating axonal growth, OPC proliferation, oligodendrocyte differentiation, remyelination and fiber tract connectivity restoration in an experimental animal model of white matter injury.

\section{Acknowledgments}

We greatly appreciate the support of Inés Barahona and Esperanza Medina and we thank ServingMed.com for linguistic assistance.

\section{Sources of funding}

Supported by research grants PS12/01754 (P.I.: EDT), INVICTUS Spanish Neurovascular Network RD12/0014/0006 (BRF and JRC) and Sara Borrell postdoctoral 
fellowship CD12/00706 (LOO) from the Research Institute Carlos III, Ministry of Science and Innovation of Spain.

\section{Disclosure}

The authors declare no conflicts of interest 


\section{References}

1. Sozmen EG, Hinman JD, Carmichael ST. Models that matter: white matter stroke models. Neurotherapeutics. 2012; 2:349-358.

2. Matute C, Domercq M, Perez-Samartin A, Ransom BR. Protecting white matter from stroke injury. Stroke. 2013; 44:1204-1211.

3. Mattson MP, Maudsley S, Martin B. BDNF and 5-HT: a dynamic duo in age-related neuronal plasticity and neurodegenerative disorders. Trends Neurosci. 2004; 27:589594.

4. Schabitz WR, Steigleder T, Cooper-Kuhn CM, Schwab S, Sommer C, Schneider A et al. Intravenous brain-derived neurotrophic factor enhances poststroke sensorimotor recovery and stimulates neurogenesis. Stroke. 2007; 38:2165-2172.

5. Schabitz WR, Berger C, Kollmar R, Seitz M, Tanay E, Kiessling M et al. Effect of brain-derived neurotrophic factor treatment and forced arm use on functional motor recovery after small cortical ischemia. Stroke. 2004; 35:992-997.

6. Xiao J, Wong AW, Willingham MM, van den Buuse M, Kilpatrick TJ, Murray SS. Brain-derived neurotrophic factor promotes central nervous system myelination via a direct effect upon oligodendrocytes. Neurosignals. 2010; 18:186-202.

7. Cellerino A, Carroll P, Thoenen H, Barde YA . Reduced size of retinal ganglion cell axons and hypomyelination in mice lacking brain-derived neurotrophic factor. Mol Cell Neurosci. 1997; 9:397-408.

8. Vondran MW, Clinton-Luke P, Honeywell JZ, Dreyfus CF. BDNF+/- mice exhibit deficits in oligodendrocyte lineage cells of the basal forebrain. Glia. 2010; 58:848-856.

9. Fisher M, Feuerstein G, Howells DW, Hurn PD, Kent TA, Savitz SI et al. Update of the stroke therapy academic industry roundtable preclinical recommendations. Stroke. 2009; 40:2244-2250. 
10. Britton GL, Kim H, Kee PH, Aronowski J, Holland CK, McPherson DD et al. In vivo therapeutic gas delivery for neuroprotection with echogenic liposomes. Circulation. 2010; 122:1578-1587.

11. Gutiérrez-Fernández M, Rodríguez-Frutos B, Ramos-Cejudo J, Vallejo-Cremades MT, Fuentes B, Cerdán S et al. Effects of intravenous administration of allogenic bone marrow- and adipose tissue-derived mesenchymal stem cells on functional recovery and brain repair markers in experimental ischemic stroke. Stem Cell Res Ther. 2013; 4:11.

12. Rogers DC, Campbell CA, Stretton JL, Mackay KB. Correlation between motor impairment and infarct volume after permanent and transient middle cerebral artery occlusion in the rat. Stroke. 1997;28:2060-2065.

13. Poduslo JF, Curran GL. Permeability at the blood-brain and blood-nerve barriers of the neurotrophic factors: NGF, CNTF, NT-3, BDNF. Brain Res Mol Brain Res. 1996; $36: 280-286$.

14. Horch HW. Local effects of BDNF on dendritic growth. Rev Neurosci. 2004; 15:117-129.

15. Goldberg MP, Ransom BR. New light on white matter. Stroke. 2003; 34:330-332.

16. Blasi F, Wei Y, Balkaya M, Tikka S, Mandeville JB, Waeber C et al. Recognition memory impairments after subcortical white matter stroke in mice. Stroke. 2014; 45:1468-1473.

17. Baumann N, Pham-Dinh D. Biology of oligodendrocyte and myelin in the mammalian central nervous system. Physiol Rev .2001; 81:871-927.

18. Back SA, Riddle A, McClure MM. Maturation-dependent vulnerability of perinatal white matter in premature birth. Stroke. 2007; 38:724-730.

19. Baracskay KL, Kidd GJ, Miller RH, Trapp BD. NG2-positive cells generate A2B5positive oligodendrocyte precursor cells. Glia. 2007; 55:1001-1010. 
20. Sommer I, Schachner M. Monoclonal antibodies (O1 to O4) to oligodendrocyte cell surfaces: an immunocytological study in the central nervous system. Dev Biol. 1981; $83: 311-327$.

21. Cui QL, D'Abate L, Fang J, Leong SY, Ludwin S, Kennedy TE et al. Human fetal oligodendrocyte progenitor cells from different gestational stages exhibit substantially different potential to myelinate. Stem Cells Dev. 2012; 21: 1831-1837.

22. Du Y, Fischer TZ, Lee LN, Lercher LD, Dreyfus CF. Regionally specific effects of BDNF on oligodendrocytes. Dev Neurosci. 2003; 25:116-126.

23. Cheatwood JL, Emerick AJ, Schwab ME, Kartje GL. Nogo-A expression after focal ischemic stroke in the adult rat. Stroke. 2008;39:2091-2098.

24. Huang EJ, Reichardt LF. Trk receptors: roles in neuronal signal transduction. Annu Rev Biochem. 2003; 72:609-642.

25. Cai D, Shen Y, De Bellard M, Tang S, Filbin MT. Prior exposure to neurotrophins blocks inhibition of axonal regeneration by MAG and myelin via a cAMP-dependent mechanism. Neuron. 1999; 22:89-101. 


\section{Figure legends}

Figure 1. BDNF levels in the brain and peripheral serum are augmented at $4 \mathrm{~h}$ after systemic BDNF administration returning to lower levels at 7 and 28d. (A). ELISA (B). Western blot and (C) Immunofluorescence (IHF) (Data are mean \pm SEM, scale bars $=20$ $\mu \mathrm{m} ; *=\mathrm{p}<.05,4$ animals, 4 sections each per group in IHF).

Figure 2. Improved functional recovery and connectivity in BDNF treated animals. (A) beam walking test (left), rotarod test (middle) and Modified Rogers Test (right). (B). Morphological study by CryoMyelin staining identified the zone of the lesion as an area of white matter injury located in the subcortical zone, showing restored myelinated axons in the BDNF-treated animals. Quantification of mean ROI intensity of the CryoMyelin staining Stroked line indicates ROI; Yellow line indicates a representative longitudinal profile of pixel intensity. (C) Qualitative and quantitative analysis of T2weighted MRI (left), axial diffusivity in DTI tractography (center) and diffusion in ADC images (right) at $28 \mathrm{~d}$ showed a significant progressive reduction in white matter injury, showing augmented connectivity of the fiber tracts in the BDNF-treated animals compared with the control group. Zoomed lesion site 3D views of DTI tractography images are shown (Data are mean \pm SEM, scale bars $=20 \mu \mathrm{m},{ }^{*}=\mathrm{p}<.05,6$ animals, 10 sections each per group).

Figure 3. Aumented cell proliferation after BDNF treatment at 7d. (A) Qualitative and quantitative analysis of cell proliferation by KI-67 staining. (B) KI-67 co-labeling with CNPase, A2B5, APC and PDGFR-alpha markers at 7d after treatment. (Data are mean \pm SEM, scale bars $=20 \mu \mathrm{m}, *=\mathrm{p}<.05,6$ animals, 10 sections each per group) 
Figure 4. Increased OPC-related markers (A2B5, CNPase, O4) at 7d after BDNF treatment. At $28 \mathrm{~d}$, all these levels were decreased compared with $7 \mathrm{~d}$ measured by immunofluorescence (A) and Western blot (B) (Data are mean \pm SEM, scale bars $=20$ $\mu \mathrm{m},{ }^{*}=\mathrm{p}<.05,4$ animals, 4 sections each per group).

Figure 5. Increased white matter maturation and axonal growth-associated markers at 28d (MBP, Olig-2) and decreased levels of Nogo-A after BDNF treatment. (A) Immunofluorescence and (B) Western blot (Data are mean \pm SEM, scale bars $=20 \mu \mathrm{m}$, $*=\mathrm{p}<.05,4$ animals, 4 sections each per group). 
Figure 1

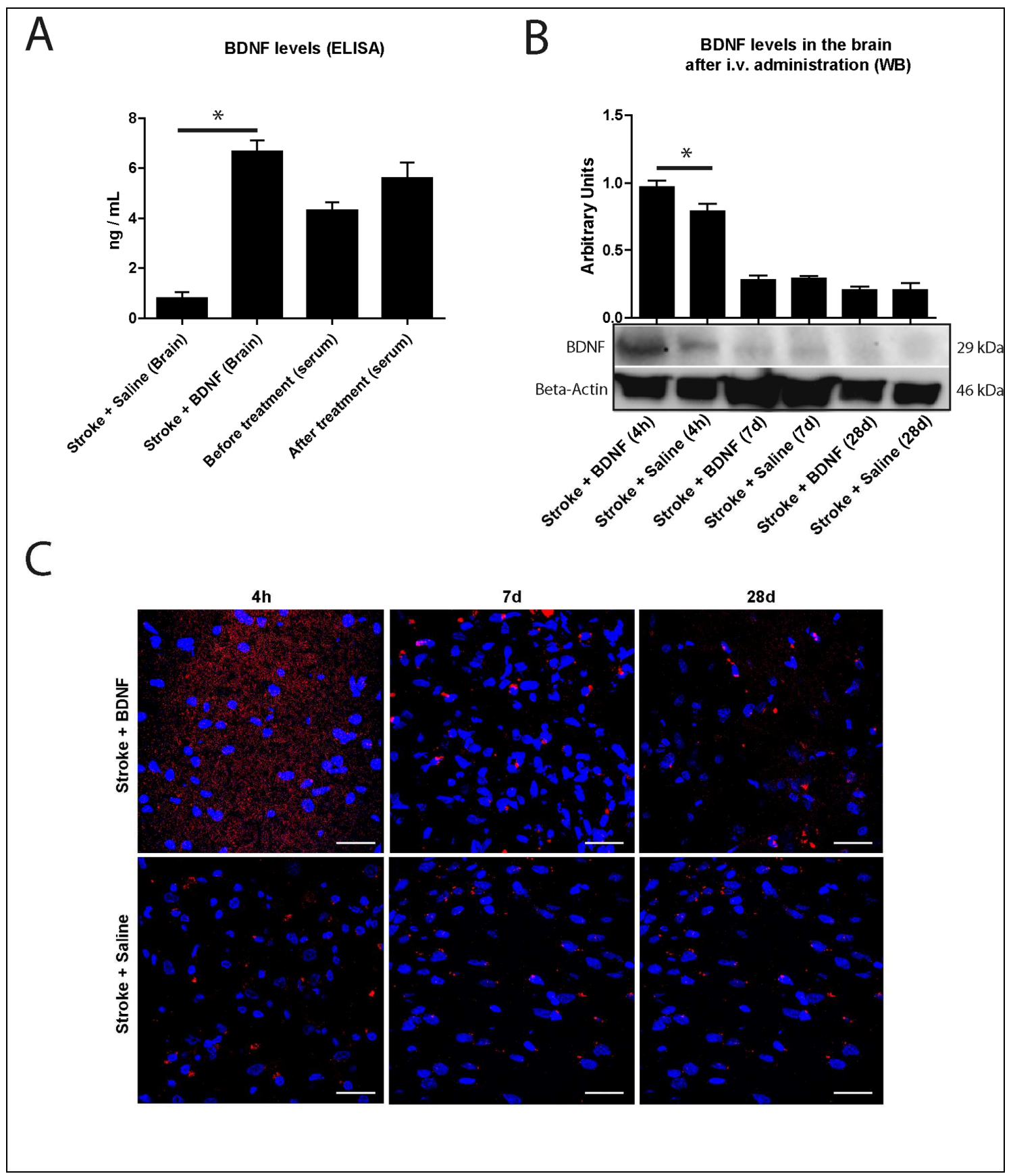


Figure 2

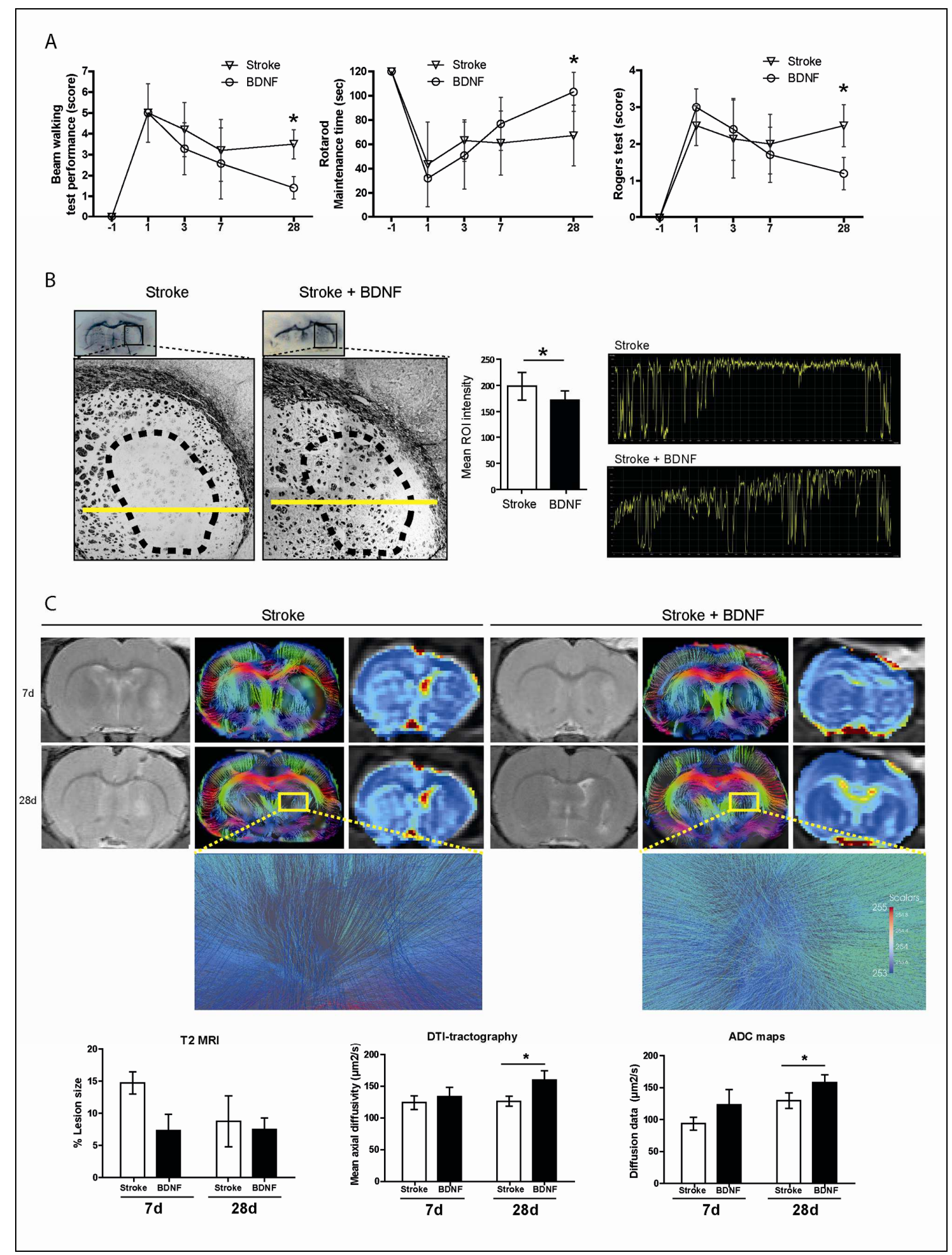


Figure 3

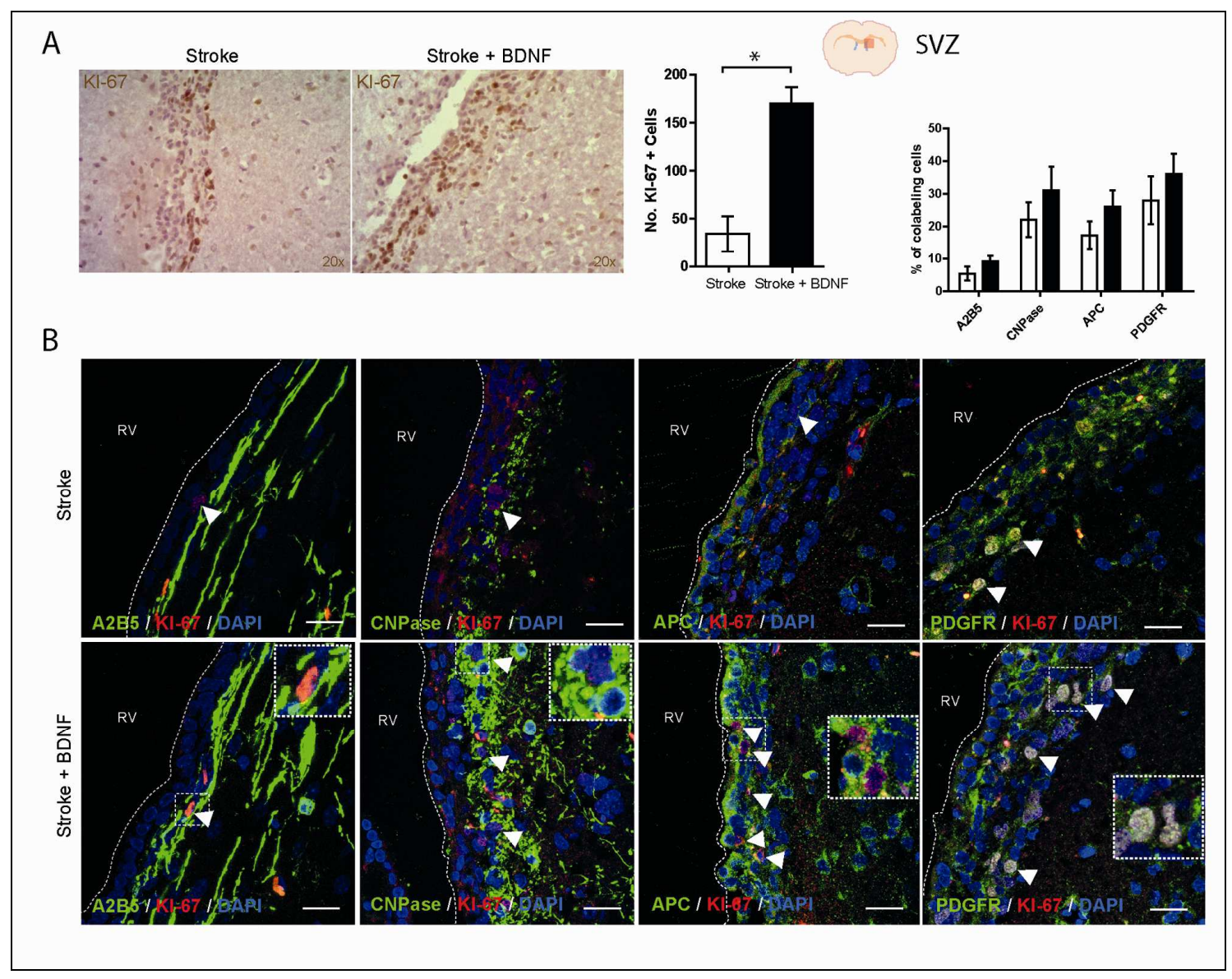


Figure 4

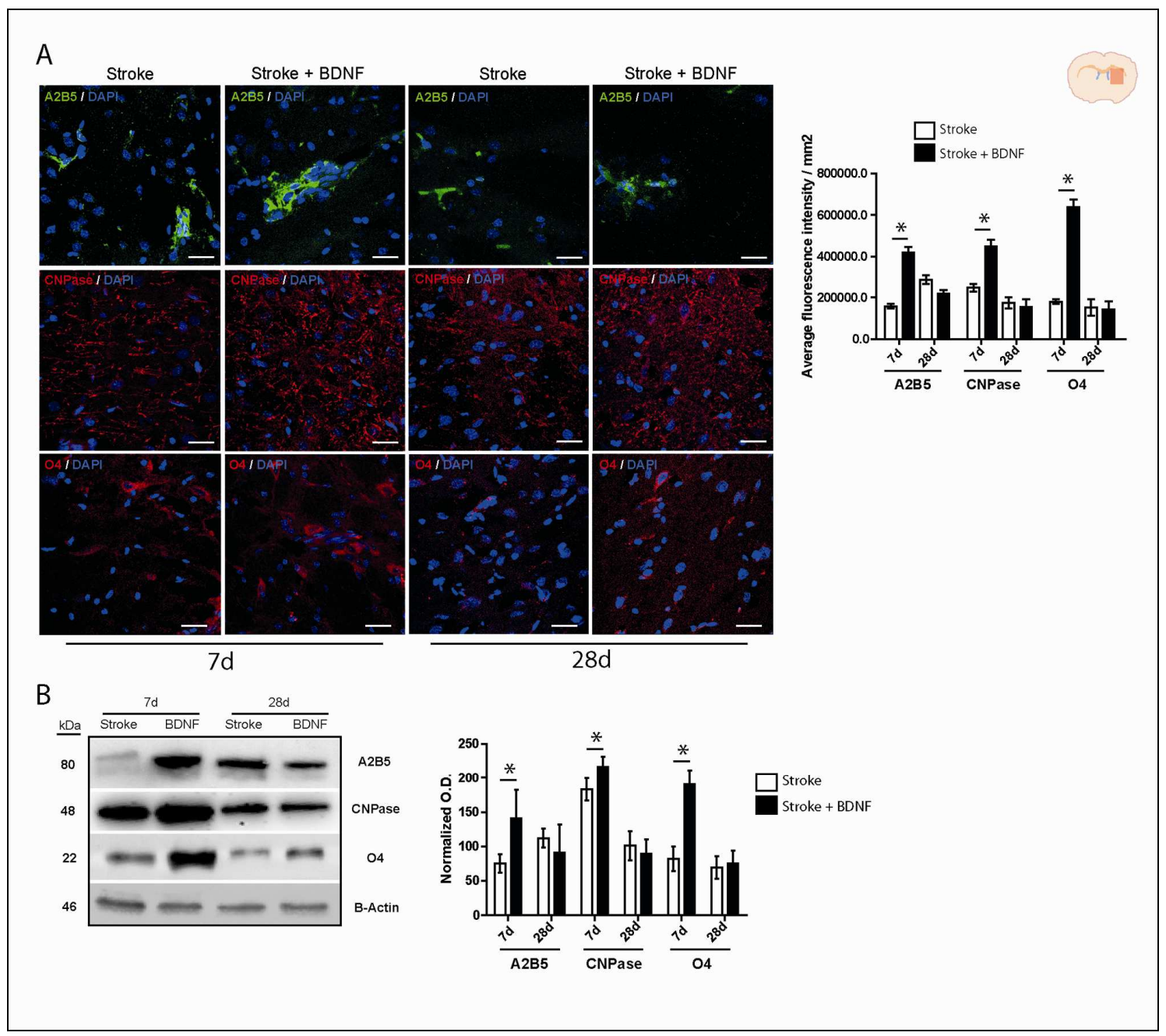


Figure 5

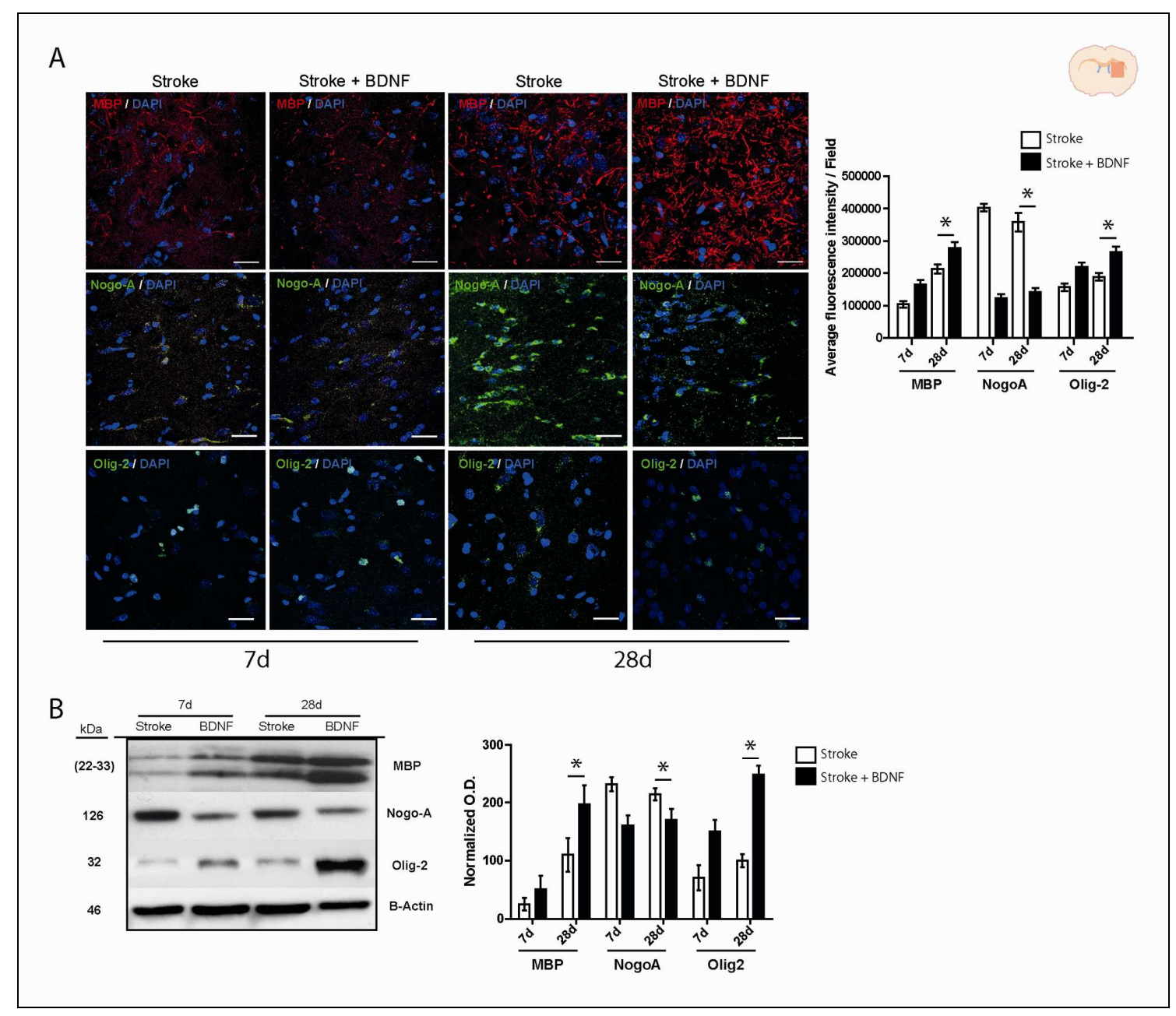




\section{SUPPLEMENTAL MATERIAL}

\section{ET-1 subcortical stroke model}

In all the animals, continuous monitoring of physiological parameters (glycemia, blood gases and blood pressure) was performed during surgery and ischemia (Monitor Omicron ALTEA RGB medical devices, Madrid, Spain). Body temperature was also monitored and maintained at $36.5 \pm 0.5^{\circ} \mathrm{C}$. All the surgical experiments were performed on adult rats that were secured on a stereotactic apparatus under isoflurane inhalatory anesthesia.

\section{BDNF quantification after treatment}

The BDNF levels were quantified by a human BDNF ELISA kit (ABCAM), Western blot and immunohistochemistry in the brain tissue and serum $4 \mathrm{~h}$ after treatment in both the control animals and the treated animals. The BDNF ELISA determination was performed according to the manufacturer's specifications. The standard curves and the samples were run in duplicate. The serum was diluted 1:20. For BDNF biodistribution analysis in the brain, $40 \mu \mathrm{g}$ of total protein from all the different isolated brain areas were added to the different ELISA sample wells. The absorbance was measured at 450nm with an Epoch microplate spectrophotometer (Bioteck). To analyze BDNF brain levels at different times, Western blot and immunofluorescence were performed $4 \mathrm{~h}, 7$ and $28 \mathrm{~d}$ after injection in the lesion zone, using a primary antibody anti-BDNF $(1: 1000$, Millipore) and a secondary antibody anti-rabbit Alexa Fluor 594 (1:750, Invitrogen). The slices were examined using a LEICA TCS SPE spectral confocal microscope (Leica Microsystems, Heidelberg, Germany) and the confocal images were analyzed using LEICA software LAS AF, version 2.0.1 Build 2043. All the images were acquired as a maximum confocal projection. For the Western blot, the units were normalized based on beta-actin levels (1:400, Sigma-Aldrich).

\section{Functional evaluation scales}

Functional evaluation was scored by a blinded observer on all the animals before surgery and at 1, 3, 7 and $28 \mathrm{~d}$ after treatment by 3 different tests. First, the beam walking test measured the ability of rats to cross a wooden beam $(2.5 \times 2.5 \times 80 \mathrm{~cm})$. Scores were assigned as follows: score 0 , traversed the beam with no foot slip; score 1 , traversed with grasping of the lateral side of the beam; score 2, showed difficulty crawling across the beam but was able to traverse; score 3, required $>10$ seconds to traverse the beam because of difficulty in walking; score 4, unable to traverse the beam; score 5, unable to move the body or any limb on the beam; and score 6, unable to stay on the beam for $>10$ seconds. Second, the rotarod test measured the latency to fall from a rotating cylinder. Beginning three days before surgery, rats were trained on the accelerating (4 to $40 \mathrm{rpm}$ ) cylinder consisting of three sessions per day. The time each animal remained on the rotarod was measured twice per animal with a 15-minutes interval between each trial. The mean of the two trials was calculated. Third, a variant of the Modified Rogers Scale consisted of a 7-point behavioral rating scale: score 0, no functional deficit; score 1, failure to extend left forepaw fully; score 2, decreased grip of the left forelimb while tail gently pulled; score 3, spontaneous movement in all directions, contralateral circling only if pulled by the tail; score 4, circling or walking to 
the left; score 5, walked only when stimulated; score 6, unresponsive to stimulation with a depressed level of consciousness; and score 7, dead.

\section{T2-MRI and DTI tractography}

The BDNF effects were studied with in vivo imaging techniques, using T2-MRI and DTI tractography. First, the lesion size was analyzed at 7 and $28 \mathrm{~d}$ after treatment using magnetic resonance imaging (MRI) (Bruker Pharmascan, Ettlingen, Germany, 7 Tesla horizontal bore magnets) and T2 maps (RARE $8 \mathrm{~T} 2,180^{\circ}$ flip angle, 3 averages) as previously described. ${ }^{1}$ The lesion area was then expressed as a percentage of the contralateral hemisphere, after correcting for brain edema. Second, for diffusion analysis, we used ADC maps. Third, for tractography, diffusion tensor data (DTI) were acquired at 7 and $28 \mathrm{~d}$ after treatment with a spin echo single shot echo planar imaging (EPI) pulse sequence using the following parameters: TR/TE: 5000/35ms; a signal average of 10, a 30 noncolinear diffusion gradient scheme with diffusion weighting of $\mathrm{b}=1000 \mathrm{~s} / \mathrm{mm} 2$ and $\mathrm{b}=0 \mathrm{~s} / \mathrm{mm} 2$, and field of view $3.5 \times 3.5 \mathrm{~cm}$. There were a total of 496 slices, and the data was acquired using 30 directions. The data were acquired with a single shot EPI sequence on a 96x96 matrix, and zero-filled k-space to construct a $128 \times 128$ image matrix. The images were obtained with medInria, a multi-platform medical image processing and visualization software. Analysis of ADC and DTItractography data was performed in the lesion zone using $n=6$ animals per group. Zoomed lesion site 3D views of DTI tractography images are represented using ParaView 4.1.0 software.

\section{Cell proliferation analysis}

For the KI-67 staining, tissue slices were placed in a boiled citrate buffer ( $\mathrm{pH} \mathrm{6)}$ and a non-specific binding was blocked for $1 \mathrm{~h}$ with $3 \%$ normal goat serum (Vector Laboratories). Ki-67 was used as a primary antibody (1:100, Millipore), and the Vectastain Universal Quick Kit PK8800 was used as a secondary antibody (1:100, Vector Laboratories). The images were acquired using a $40 \times$ objective lens and the total number of positive cells were counted in a minimum of 10 different microscope fields using image analysis software.

To study the differentiation of the proliferating cells, we double stained KI-67-positive cells (1:100, Millipore) with 2',3'-Cyclic-nucleotide3'-phosphodiesterase (CNPase) (1:500, Sigma-Aldrich), A2B5 (1:500, Millipore), APC (CC1) (1:20, Abcam) and PDGFR-alpha (1:100, Abcam). The corresponding secondary antibodies were goat antimouse Alexa Fluor 488 and Alexa Fluor 594 (1:750, Invitrogen). The co-stained cells were observed using the $40 \times$ objective lens with LEICA software LAS AF. All the images were acquired as a maximum confocal projection.

\section{Immunohistochemistry, immunofluorescence and Western blot}

The staining of the myelin and myelinated axons was performed on frozen sections using a CryoMyelin kit (Hito Biotech, USA), which allows sensitive localization and visualization of the myelin fibers. The lesion zone was studied in more detail using immunofluorescence and Western blot, as previously described $(n=4$ animals of each group). ${ }^{1}$ The different primary antibodies used for immunofluorescence and Western blot analysis were CNPase (1:500, Sigma-Aldrich), A2B5 (1:500, Millipore), Oligodendrocyte marker-4 (O4) (1:25, Millipore), Nogo-A (1:100, ABCAM), myelin 
basic protein (MBP) (1:100, ABCAM) and the marker oligodendrocyte marker-2 (Olig2) (1:500, Millipore). The secondary antibodies for immunofluorescence were goat antimouse Alexa Fluor 488 and anti-rabbit Alexa Fluor 594 (1:750, Invitrogen). The slices were examined using a LEICA TCS SPE spectral confocal microscope (Leica Microsystems, Heidelberg, Germany) and the confocal images were analyzed using LEICA software LAS AF, version 2.0.1 Build 2043. All the images were acquired as a maximum confocal projection. To quantify the levels of brain markers by immunofluorescence, the experiments, images and quantification of the control and the BDNF samples were performed the same day, with the same microscope configurations and by blinded observers to eliminate bias due to background normalization. For the Western blot, the units were normalized based on beta-actin levels (1:400, SigmaAldrich).

\section{References}

1. Gutiérrez-Fernández M, Rodríguez-Frutos B, Ramos-Cejudo J, Vallejo-Cremades MT, Fuentes B, Cerdán S et al. Effects of intravenous administration of allogenic bone marrow- and adipose tissue-derived mesenchymal stem cells on functional recovery and brain repair markers in experimental ischemic stroke. Stem Cell Res Ther. 2013; 4:11. 